\title{
On the time variability of the Evershed effect
}

\author{
L. H. M. Rouppe van der Voort ${ }^{\star}$
}

\author{
The Institute for Solar Physics of the Royal Swedish Academy of Sciences, AlbaNova University Center, 10691 Stockholm, \\ Sweden
}

Received 9 September 2002 / Accepted 14 October 2002

\begin{abstract}
The time variability of the Evershed effect in the penumbra of a sunspot is studied from a time series of high-spatial resolution spectrograms covering the wings of the Ca II K line accompanied by Ca II K slit-jaw images obtained with the Swedish Vacuum Solar Telescope. Simultaneous G-band observations from the Dutch Open Telescope are used to study the penumbral scene around the spectrograph slit on the photospheric level. Four spectral lines are selected to probe the Evershed effect at different heights in the penumbral atmosphere. The Evershed flow is found to show variations on a time scale of 8-14 min which is a confirmation of earlier observations from tunable filter instruments. The largest amplitudes in the variation are found where the Evershed flow is strongest. No systematic height dependence is found for the propagation of the velocity variations. On several occasions, an increase in the Evershed signal is associated with an intensity increase. In the weak Evershed signal detected in bright filaments, no significant variability is found. As an alternative to the interpretation of temporal enhancements in the flow speed, it is speculated that temporal height variations of a flow channel hosting a steady Evershed flow can explain the observed variations in the Evershed effect.
\end{abstract}

Key words. sunspots - Sun: photosphere

\section{Introduction}

The Evershed effect (Evershed 1909) is the dominant process that gives penumbral spectral lines their characteristic line profiles and plays a central role in studies of the sunspot penumbra. It is typically observed as Doppler-shifted and asymmetric spectral line profiles and is commonly interpreted as an outflow of gas directed along penumbral filaments (for recent reviews see Muller 1992; Thomas 1994; Maltby 1997; Wiehr 1999). The observational characteristics are still not fully understood and with the advancement of solar telescopes and observational techniques, new aspects are being found.

From high-spatial resolution observations with tunable filter instruments, it was observed that the Evershed flow is not a steady flow (Shine et al. 1990), but displays an irregular repetitive behaviour (Shine et al. 1994; Rimmele 1994). Movies of Dopplergrams revealed moving structures of enhanced Doppler signal moving radially outwards in the penumbra. These velocity packages, also called "Evershed clouds", have a radial extent of about $1000 \mathrm{~km}$ and are radially separated by $2000-3000 \mathrm{~km}$. The pattern of moving velocity packages was found to have a quasi-periodic variability on a time scale of 10-15 min. The modulation can have a peak-to-peak amplitude of $1 \mathrm{~km} \mathrm{~s}^{-1}$, superimposed on an average steady flow of 3-4 $\mathrm{km} \mathrm{s}^{-1}$ (Shine et al. 1994).

Two models of the Evershed effect are most successful in explaining a number of observational properties: the siphonflow model as proposed by Montesinos \& Thomas (1997), and

\footnotetext{
^ e-mail: rouppe@astro.su.se
}

the moving-tube model by Schlichenmaier et al. (1998a,b). In the siphon-flow model, magnetic loops act as siphons where the gas flow is driven by an unbalanced gas pressure due to a difference in magnetic field strength between the foot-points. In the numerical simulations of the moving-tube model, penumbral filaments are modelled as thin magnetic flux tubes in a superadiabatic and magnetized penumbral atmosphere. As a tube convectively rises to the surface, an outflow is built up which is identified as the Evershed flow. Neither of the two models explains the observed time variability of the Evershed effect, although recent progress has been made (Schlichenmaier 2002).

In this paper, I present the first study on the time variability of the Evershed flow that employs a spectrograph, so that complete spectral line profiles are measured directly. This excludes the possibility of the Doppler measurements being contaminated by seeing effects.

\section{Observations}

The leader sunspot in NOAA Active Region 8704 was observed on 20 September $1999\left(\mathrm{~S} 19.2^{\circ}, \mathrm{E} 31.5^{\circ}, \mu=0.77\right)$ with the Swedish Vacuum Solar Telescope (SVST) on La Palma (Scharmer et al. 1985). Spectra of the Ca II K line (3933.66 ^) were obtained with the short Littrow spectrograph (Scharmer et al. 1985), spanning a wavelength interval of $16.7 \AA$ (starting at $3923.7 \AA$ ) which covers a significant fraction of the extended $\mathrm{Ca}$ II $\mathrm{K}$ damping wings. Operating with a $25 \mu \mathrm{m}(0.23)$ wide slit, the spectral resolution was slit-limited corresponding to a bandpass of $30 \mathrm{~m} \AA$ at the wavelength of Ca II K. The spectrograph camera recorded spectrograms with an exposure 

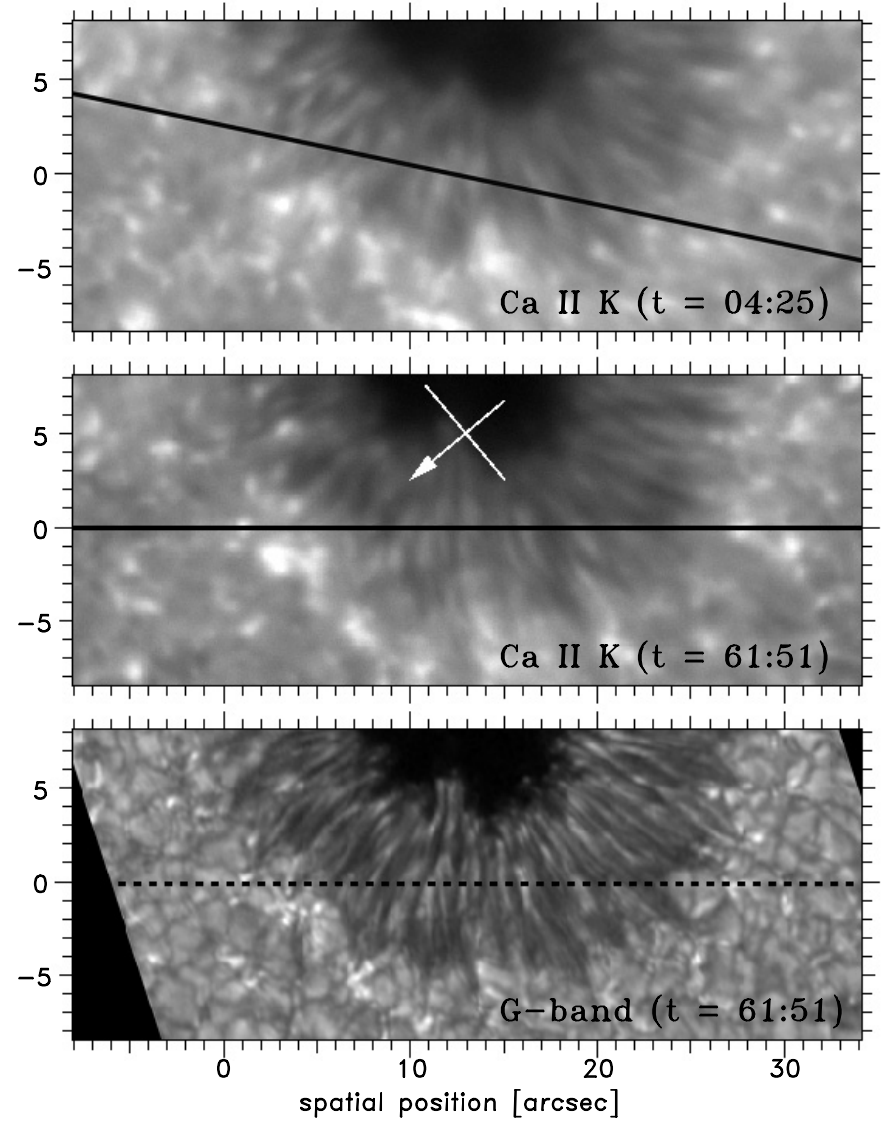

Fig. 1. Two Ca II K slit-jaw images, one from the beginning (top) and one from the end of the sequence, and one G-band image (DOT). The black lines drawn in the slit-jaw images mark the location of the spectrograph slit. Note the change in slit angle due to image rotation. The approximate location of the slit in the G-band image is marked with the dashed line. The white arrow in the central image points towards disk centre.

time of $0.35 \mathrm{~s}$ and operated at a pixel scale that corresponded

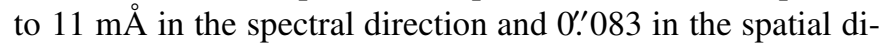
rection. The exact location of the spectrograph slit on the Sun was determined from slit-jaw images recorded with a narrow (3 A bandpass) interference filter centered on the Ca II K line core. In order to assure an accurate correspondence between the spectrogram and the associated slit-jaw images, the exposures were recorded strictly simultaneously using synchronized CCD cameras. Photospheric reference images were recorded independently with a $12 \AA$ bandpass interference filter centered on the G-band (4305 $\AA$ ).

The time series discussed in this article span almost $64 \mathrm{~min}$, starting at 13:47 UT. The spectrograph slit covered the penumbra on the disk-centre side of the sunspot, as illustrated in the slit-jaw images in Fig. 1. The penumbral filaments that were crossed by the spectrograph slit around spatial location $=20^{\prime \prime}$, were oriented perpendicularly to the line-of-sight. No Evershed effect was observed in this particular part of the penumbra, since the direction of the flow is along the penumbral filaments.

On-line frame selection (Scharmer \& Löfdahl 1991) was applied to select the best quality spectrogram from a series of images taken in a $18.5 \mathrm{~s}$ interval, resulting in a total of
207 spectrogram/slit-jaw image pairs. The correlation tracker (Shand et al. 1995) stabilized the images to a residual image jitter of less than 0.'5 rms, with a maximum of less than $1^{\prime \prime}$. In order to keep the spectrograph slit covering the same penumbral features during the time series, the telescope was operating in a 3-step scanning mode. Between frame selection intervals in a scan cycle, the image was moved one slit width (0.'23) perpendicularly to the slit direction. After selecting the third spectrogram, a new cycle was begun at the starting position.

The altaz mount of the telescope turret introduced an image rotation of $13^{\circ}$ between the first and last frame, with the rotation axis centered in the umbra (see Fig. 1). This resulted in a drift with respect to the spectrograph slit, with a magnitude depending on the distance from the rotation axis. In the penumbra, the maximum drift was less than $3^{\prime \prime}$ across the slit, and about 2 ". 5 along the slit.

The seeing conditions at La Palma on 20 September 1999 were very favourable and part of the SVST observations obtained that day are discussed in Rouppe van der Voort (2002, hereafter Paper I) to which the reader is referred for more details on the observations and instrumentation.

The wings of the Ca II K line were selected for its broad depth-range in temperature sensitivity and the presence of a large number of isolated spectral line blends with varying line strengths that can be used as velocity diagnostics. Like described in Paper I, inversions of the Ca II $\mathrm{K}$ wings yield information on the temperature stratification of the atmosphere under the spectrograph slit, while the combined measurements of line-core Doppler shifts provide information on velocity gradients. However, this paper focuses on the velocity measurements of the line blends only.

During part of the time interval (starting from 14:10 UT), the Dutch Open Telescope (DOT, Hammerschlag \& Bettonvil 1998) observed the same sunspot with a G-band filter. This frame-selected filtergram series has a cadence of $20.3 \mathrm{~s}$, which matches the spectrogram cadence more closely than the SVST G-band series (frame rate was 1 per $60 \mathrm{~s}$ ) and was included in this work to study the photospheric penumbral scene around the location covered by the spectrograph slit. More details about the DOT observations can be found in Sobotka \& Sütterlin (2001) and Balthasar et al. (2001).

\section{Data processing}

Data reduction and analysis were performed using the data processing software package and language ANA (Shine 1990).

\subsection{Spectra and filtergrams}

The reduction of the spectrograms was performed using standard procedures, including flatfielding, wavelength calibration and noise reduction using Fourier filtering techniques (more details in Paper I).

The slit-jaw images were first flatfielded and then rescaled to match the spatial size of spectrogram pixel. The alignment to the spectrograms was done by cross-correlation of two spatial intensity profiles of the area covered by the slit. The slit is etched on a Cr-coated glass plate, so that besides being 
transmitted into the spectrograph, part of the light falling on the slit is being reflected by the glass into the slit-jaw camera beam. The spatial intensity profile under the slit could therefore be recovered from the slit-jaw images and be correlated for alignment with the corresponding spatial intensity profile from the spectrograms, derived from the convolution of the spectrograms with the transmission profile of the slit-jaw filter.

Finally, a movie was produced in order to study the time evolution of the solar scene around the slit. The movie frames were stabilized using cross-correlation to a reference image, correcting for image jitter induced by seeing and the scanning mode. No destretching was applied to the slit-jaw images since that would introduce a deformation of the straight slit in the image domain.

From the alignment of the slit-jaw images, the exact offsets of the spectrograph slit could be determined. From each 3-step scan cycle, one spectrogram/slit-jaw image pair was selected so that in the resulting series, the spectrograph slit had minimum relative offsets. The selected series has a maximum jitter in the slit position of less than 2 slit-widths and a cadence of $55.5 \mathrm{~s}$.

The reduction of the DOT images include flatfielding, rigid alignment, destretching and correction for the instrumental and atmospheric MTF (more details in Balthasar et al. 2001).

The SVST G-band images were manually aligned to the slit-jaw images after flatfielding and rescaling the pixel dimensions. These images were used to facilitate a solid alignment of the DOT G-band images to the SVST observations. A pixelto-pixel alignment of the DOT images to the slit-jaw images was not attempted to be achieved but the alignment is accurate enough to determine the slit location at the photospheric level.

\subsection{Doppler- and intensitygrams}

From the set of spectral lines present as blends in the $\mathrm{Ca}$ II $\mathrm{K}$ wings, a subset was selected to study the Evershed effect at different heights in the penumbral atmosphere. These spectral lines are well isolated and relatively strong, Table 1 presents the line core wavelengths determined from a spatially-averaged disk-centre intensity solar atlas (Neckel 1999). The line core mean height of formation is determined from LTE line formation modelling in the quiet Sun atmosphere model of Holweger \& Müller (1974, HolMul hereafter). More details, including a summary of atomic data, are presented in Paper I.

The weak slope of the Ca II K wings on which the line blends are superposed, affects the shape of the spectral line profiles as compared to spectral lines with a clean continuum. Van der Waals broadening is the dominant broadening agent and the slope of the CaII $\mathrm{K}$ wings depends on the temperature stratification of the atmosphere under the slit. Since the Doppler shifts of the line blends are determined over a small portion of the line profile, variations in the slope of the $\mathrm{Ca}$ II $\mathrm{K}$ wings could in principle affect these measurements. To estimate the strength of this effect, some numerical tests were performed of Doppler measurements on synthetic lines from hot and cool penumbral atmospheres (from Paper I) with realistic velocities present. It was found that the measurement differences between the lines was on the order of less than
Table 1. Summary of spectral lines. Line core wavelength is determined from a disk-centre intensity solar atlas. Height is line-core mean height of formation above the continuum in the HolMul atmosphere model (from Paper I).

\begin{tabular}{ccc}
\hline \hline Ion & $\begin{array}{c}\text { Wavelength } \\
{[\AA]}\end{array}$ & $\begin{array}{c}\text { Height } \\
{[\mathrm{km}]}\end{array}$ \\
\hline $\mathrm{Mn}_{\mathrm{I}}$ & 3926.475 & 148 \\
$\mathrm{Fe}_{\mathrm{I}}$ & 3940.039 & 177 \\
$\mathrm{Fe}_{\mathrm{I}}$ & 3925.207 & 283 \\
$\mathrm{Ti}_{\mathrm{I}}$ & 3929.874 & 323 \\
\hline
\end{tabular}

a few $\mathrm{m} \mathrm{s}^{-1}$ - much smaller than other uncertainties involved (see below).

The Doppler shift of the line core was determined from the analytical minimum of a second order polynomial fitted to 5 pixels $(0.044 \AA)$ centered at line minimum. From a numerical experiment, the uncertainty due to noise was determined to be on the order of $120 \mathrm{~m} \mathrm{~s}^{-1}$. This uncertainty is reduced by binning the spectrograms in the spatial dimension over 3 pixels (0!'25). Random errors introduced by seeing variations can be estimated from the difference in velocity between consecutive spectrograms. For these lines, the rms of the differences in the penumbra ranges between $150-200 \mathrm{~m} \mathrm{~s}^{-1}$. Note that this is an overestimate since these differences are partly real and partly introduced by alignment errors and slit displacements.

The line-core shifts were calibrated to a reference constructed from the line core positions of the spectra well outside the penumbra. The centroid of a Gaussian fitted to the linecore shifts from a $34^{\prime \prime}$ long part of all 207 spectrograms served as reference line core position. This procedure is too biased to serve as an absolute velocity calibration: there is relatively small spatial smearing because of the rather stable slit pointing and the abnormal granulation surrounding a sunspot introduces an unknown offset with respect to standard quiet sun granulation. However, this calibration is precise enough for this study which focuses on the relative change of the Doppler signal in the penumbra.

After determining the Doppler shifts in each spectrogram, intensity and velocity space/time diagrams were constructed for each line by subsequently putting an intensity or velocity profile from each spectrogram on top of another. In such a diagram, the spatial dimension is along the horizontal axis and time runs along the vertical. The initial diagrams had a continuous spatial drift introduced by image rotation and an additional jitter from seeing variations and the scanning mode. These were largely removed by shifting each subsequent profile by an amount determined from a rigid alignment of an intensity space/time diagram in the far $\mathrm{Ca}$ II $\mathrm{K}$ wing where the intensity profile has largest contrast. The shifts determined from a cross-correlation procedure on this diagram were applied to all diagrams and the results are shown in Fig. 2. 


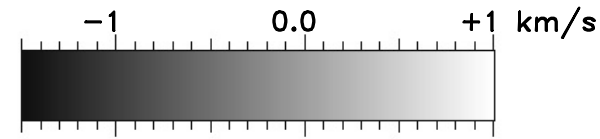

a: $M n \mid 3926 \AA$

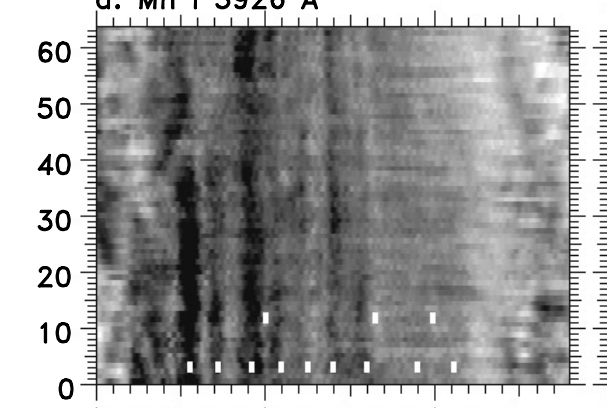

b: Fe I $3940 \AA$

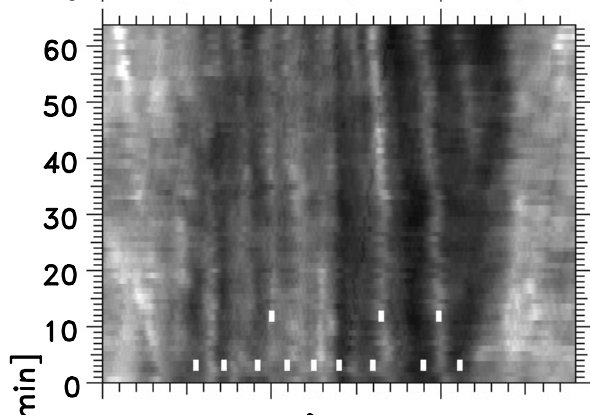

c: Fe $\mid 3925 \AA$

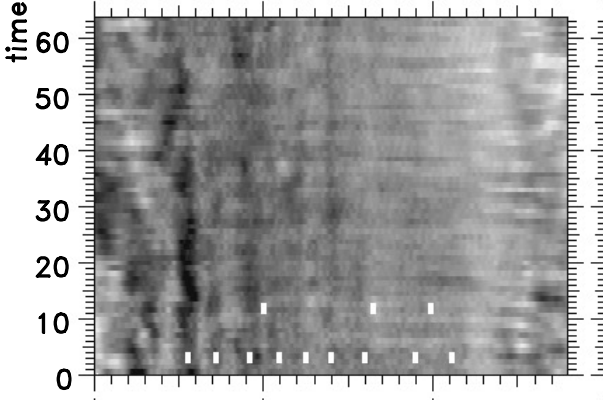

d: $\mathrm{Ti}$ । $3929 \AA$
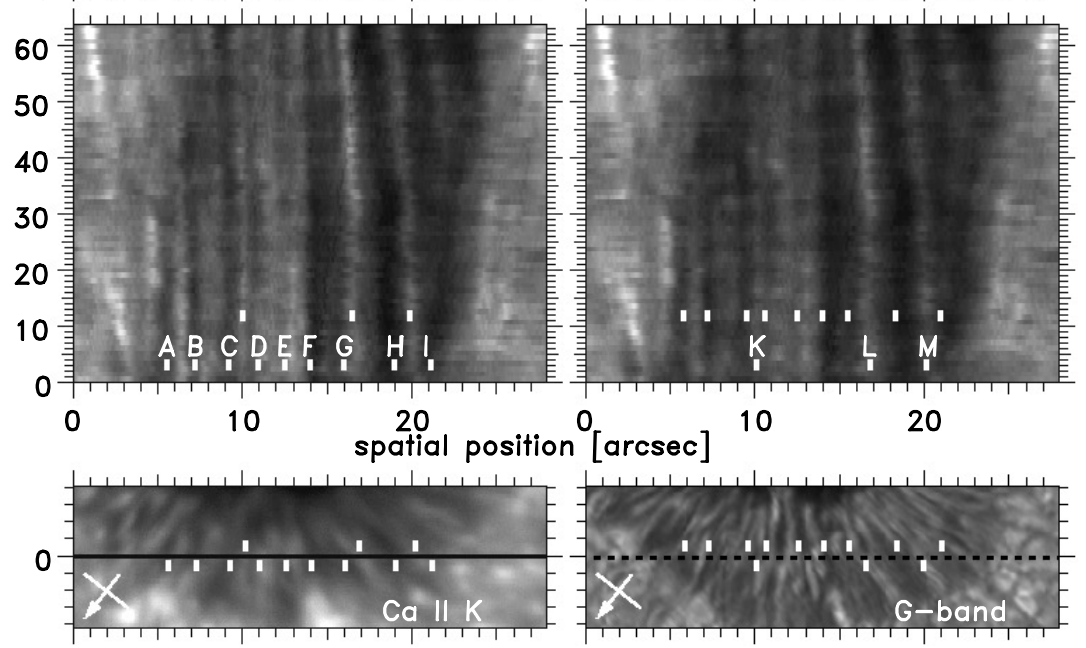

Fig. 2. Pairs of line-core intensity (bottom) and velocity (top) space/time diagrams for the four spectral lines. The Doppler diagrams are grayscaled according to the scaling box at the top left, the intensity diagrams are scaled individually for each spectral line. The spatial coordinates have the same origin as in Fig. 1. Small white rectangles mark the different filaments discussed in the text, dark filaments have letter designation $A-I$ (bottom left diagram) and bright filaments $K-M$ (bottom right). Below the space/time diagrams a $\mathrm{Ca}$ II $\mathrm{K}$ slit-jaw and a G-band image (the approximate location of the slit indicated by the dashed line) with the same filament markings. The arrow points towards disk centre.

\subsection{Tracking filaments}

The solar image under the spectrograph slit was in constant motion, by translation and distortion due to seeing effects, by image rotation from the telescope and by deliberate translation from the scanning procedure. After determination of the magnitude of the shifts and a careful inspection of the slit-jaw movie, it was concluded that a number of penumbral filaments were covered during the whole sequence. In order to study the velocity signal in each of these filaments in close detail, tracks were selected in the space/time diagrams. The algorithms to set up the different tracks were based on either following local minimum intensity (for dark filaments), maximum intensity (for bright filaments) or maximum velocity signal. Maximum 
velocity signal and minimum intensity turned out to give very similar results - reflecting the often reported observation of the strongest Evershed effect being found in dark filaments (see e.g. Beckers 1968; Title et al. 1993; Shine et al. 1994; Rimmele 1995). Each track was displayed in the slit-jaw movie to assure that the same filament was followed during the whole sequence. In all the velocity/intensity diagram pairs plus the G-band and slit-jaw images in Fig. 2, the tracks are marked with small white rectangles, with their letter designation given in the lower diagrams. Track $A$ and $B$, corresponding to the two left-most dark filaments were covered only part of the sequence, image rotation moved the spectrograph slit away from these filaments after about $40 \mathrm{~min}$.

\section{Results}

\subsection{Space/time diagrams}

Figure 2 shows the space/time diagrams for the four spectral lines. As indicated by Fig. 1, the left-hand side of the slit crossed penumbral filaments that were directed at small angles towards disk centre. Here, the Evershed signal is strongest as opposed to the right-hand side of the diagrams, where the slit crossed penumbral filaments perpendicular to the line-of-sight and the Evershed signal is virtually absent. In addition, on the left-hand side of the diagrams, the slit crosses the outer regions of the penumbra, where the Evershed effect is known to be generally stronger than for smaller radial distances from the umbra.

A close comparison between the intensitygrams and the Dopplergrams shows that the regions where the Evershed effect is strongest coincide with dark filaments. As noted in Sect. 3.3, this is a confirmation of a regularly reported observation. Note however, that the velocity signal in the bright filaments is not absent everywhere, most notably on the left side of the slit in Fig. 2 (see also Sect. 4.4). This points to a non-perfect correlation which might be the basis of a number of conflicting observations of an unclear correlation (see e.g. Wiehr \& Stellmacher 1989; Lites et al. 1990; Johannesson 1993; Hirzberger \& Kneer 2001; Paper I).

The velocity/intensity diagram pairs in Fig. 2 are ordered for increasing line-core formation height. It is easily recognized that the Evershed signal decreases in magnitude for increasing formation height, a commonly known property (see e.g. St. John 1913a,b; Rimmele 1995; Paper I).

For a number filaments, e.g. $A, B, C$ and $F$, Evershed signal is detected during the whole period when covered by the spectrograph slit. These filaments can be characterized as Evershed channels, filaments with a persistent Evershed flow.

The Dopplergrams show that the Evershed signal is not stationary, periods with stronger signals alternate with periods with weaker signals, e.g. for filament $C$, a period of strong signal ends at $t \approx 40 \mathrm{~min}$ and starts again at $t \approx 55 \mathrm{~min}$. On the right-hand side in the penumbra, where the Evershed signal is weak, the Doppler signal is dominated by $p$-mode oscillations, recognized by horizontal bright and dark bands, spatially coherent over a significant fraction of the slit.

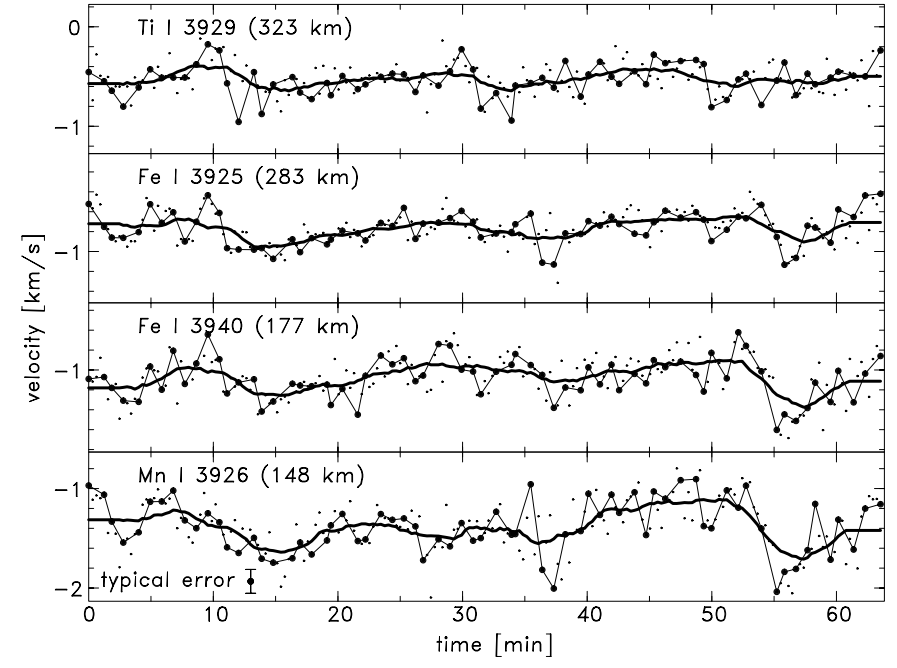

Fig. 3. Velocity signals in filament $C$ for all spectral lines. Large dots mark the selected data points from slit positions separated less than 2 slit widths from each other. The small dots mark all other data points, i.e. from slit positions with larger spatial offsets, which are omitted from further analysis. A 5.5-min boxcar smoothing is outlined by the thick solid line.

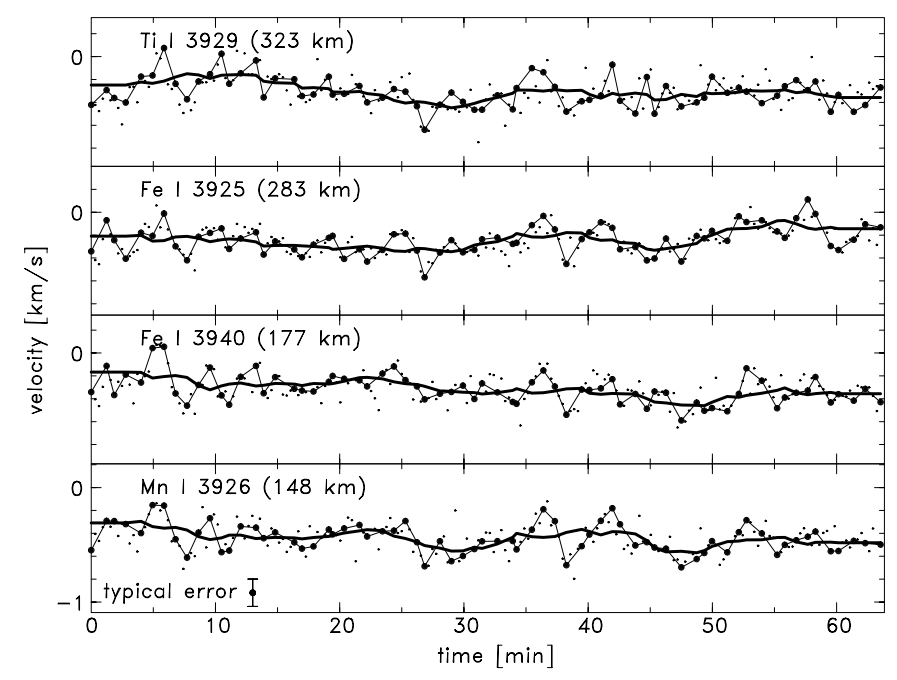

Fig. 4. Velocity signals in filament $H$ for all spectral lines.

\subsection{Velocity signal in dark filaments}

In Fig. 3, the velocity signal in filament $C$ is shown for the four spectral lines. Filament $C$ is covered by the spectrograph slit during the whole sequence and hosts a strong Evershed flow. The larger dots (connected with the thin solid lines) are data points from the selected series, slit positions that are separated less than two slit widths from each other (see Sect. 2). The power spectrum of the velocity signal (not shown) has a prominent peak around 5 min for all four lines so that on short time scales, the variability in the velocity signal is dominated by $p$ mode oscillations. The thick solid lines show the residual signal after suppression of the short-time variability by a 5.5-min boxcar smoothing which leaves a significant variability on longer time scales. The largest amplitudes are found for the lowest formed line, Mn I 3926. 


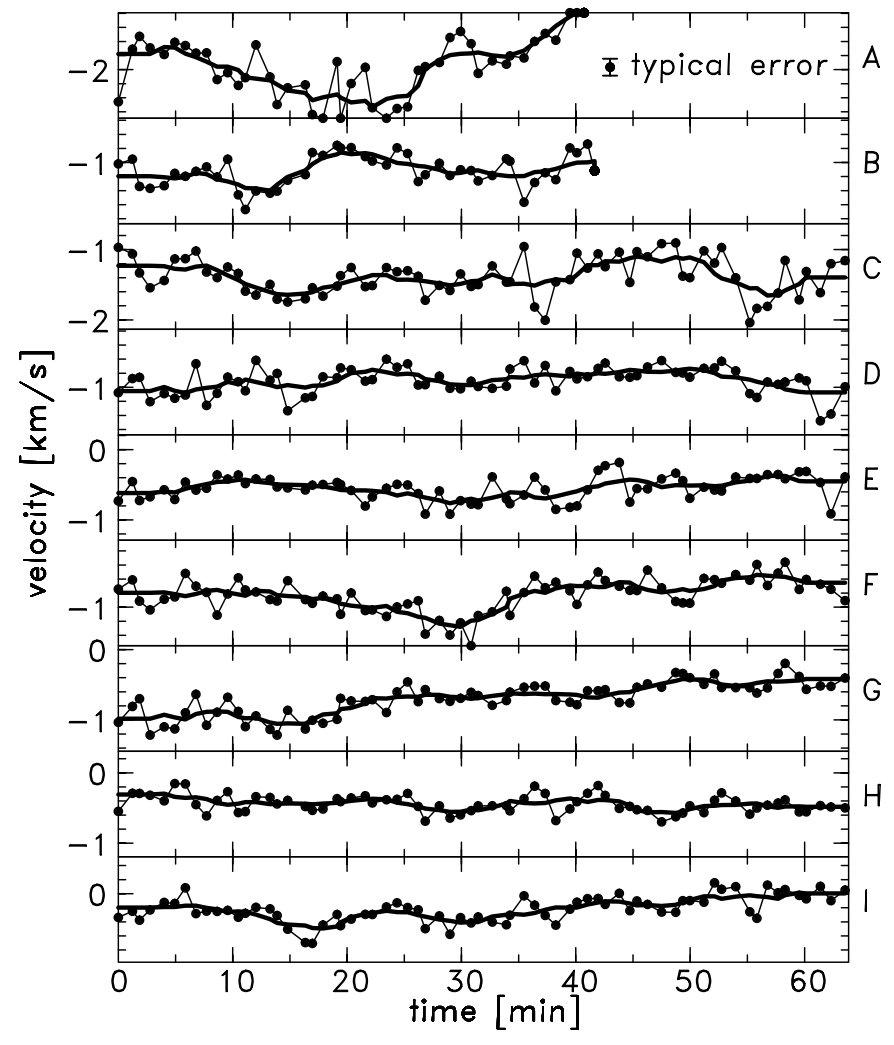

Fig. 5. Velocity signal for all filaments for Mn I 3926. The spatial ordering of the filaments can be found in Fig. 2. Filament $A$ and $B$ were only partly covered; image rotation moved the spectrograph slit away from these filaments after approximately $40 \mathrm{~min}$.

The velocity signals for filament $H$ are shown in Fig. 4. The orientation of filament $H$ is close to perpendicular to the line-of-sight and no significant Evershed effect is observed in this filament. The mean velocity offset of $\sim-100 \mathrm{~m} \mathrm{~s}^{-1}$ is well within the uncertainty of the absolute velocity calibration. Like for filament $C$, the variability on short time scales is dominated by $p$-mode oscillations, the smoothed curve does not show any significant residual variability.

The velocity signals of Mn I 3926 for all dark filaments are shown in Fig. 5. The ordering of the filaments is (see also Fig. 2) from a part where a strong Evershed signal is observed to a part where no significant Evershed signal is observed. The connected thick dots (and the power spectra) show $p$-mode contribution in all filaments, but the smoothed curves show only significant variability in the filaments with stronger Evershed signal. This time variation can be attributed to variability of the Evershed effect.

The comparison between filaments with strong Evershed signal showing long-time variability and filaments without Evershed signal, is a strong argument against any systematic effects (e.g. instrumental, in the velocity calibration etc.) or beats from higher frequency oscillations. Another argument supporting the existence of long-term Evershed variability in the data is that similar time behaviour is observed for all spectral lines. Finally, errors from noise, seeing and image jitter are estimated to be low enough for the observed Evershed variability to be real.
Table 2. Peak-to-peak velocities $\left[\mathrm{km} \mathrm{s}^{-1}\right]$ for four filaments. In each column, the measured Doppler velocity (line-of-sight component of the velocity) is given with the de-projected value between parentheses. For each filament, the first column is the velocity range of the smoothed $(5.5 \mathrm{~min})$ data, the second column is the velocity range of all (selected) data points, i.e. including $p$-mode contribution. Deprojection is performed under the assumption that the Evershed flow is horizontal and parallel to the orientation of the hosting filament.

\begin{tabular}{|c|c|c|c|c|}
\hline & \multicolumn{2}{|c|}{$A$} & \multicolumn{2}{|c|}{$B$} \\
\hline Mn I 3926 & $1.6(2.5)$ & $2.2(3.4)$ & $0.5(0.8)$ & $0.9(1.5)$ \\
\hline $\mathrm{Fe}_{\text {I }} 3940$ & $1.1(1.7)$ & $1.4(2.2)$ & $0.3(0.5)$ & $0.9(1.5)$ \\
\hline Fe г 3925 & $0.8(1.3)$ & 1.0 (1.6) & $0.3(0.5)$ & $0.5(0.8)$ \\
\hline Ті г 3929 & $0.3(0.5)$ & $1.0(1.6)$ & $0.3(0.5)$ & $0.8(1.4)$ \\
\hline & \multicolumn{2}{|c|}{$C$} & \multicolumn{2}{|c|}{ ) } \\
\hline Mn I 3926 & $0.5(0.9)$ & $1.1(1.9)$ & $0.3(0.6)$ & $0.9(1.5)$ \\
\hline $\mathrm{Fe}_{\mathrm{I}} 3940$ & $0.4(0.7)$ & $1.0(1.8)$ & $0.3(0.6)$ & $0.7(1.2)$ \\
\hline $\mathrm{Fe}_{\text {I }} 3925$ & $0.3(0.5)$ & $0.7(1.2)$ & $0.2(0.3)$ & $0.7(1.2)$ \\
\hline Ті г 3929 & $0.3(0.5)$ & $0.8(1.4)$ & $0.3(0.6)$ & $0.7(1.2)$ \\
\hline
\end{tabular}

The variations in the Evershed flow do not seem to behave in a regular oscillation with a unique amplitude or period. A typical time scale for the variations lies between 8-14 min, where the power spectra have a prominent peak. This is consistent with the time scales reported by Shine et al. (1994) (10 min) and Rimmele (1994) (15 min). Values that could characterize the velocity amplitudes of the variations are given in Table 2. Here, peak-to-peak velocities are given for the first four filaments, where the Evershed effect is strongest. The largest amplitudes in the variation seem to be found where the Evershed flow is strongest: in the lowest formed lines and in the strongest channel (filament $A$, near the outer penumbral boundary). Typically, the amplitude is on the order $40-60 \%$ of the mean Evershed flow speed.

In an attempt to search for systematic differences in the Evershed variability between the different spectral lines, the velocity plots were closely compared. No significant time delays in the variations were found so that it seems that there are is no systematic height dependence for the propagation of the velocity variations.

\subsection{Velocity-intensity correlation}

Shine et al. (1994) found a positive correlation between the Evershed Doppler clouds and continuum intensity: an increase in the Evershed signal was observed to be usually associated with a relative increase in the continuum intensity. Figure 6 shows a comparison for four filaments between intensity in the far Ca II $\mathrm{K}$ wing (formed only just above the continuum forming layers, $\log \left(\tau_{5000}\right) \approx 0.05$ in HolMul for $\lambda=3924 \AA$, see Paper I) and velocity in Mn 3926 (Note that for these filaments in the centre-side penumbra, an increasing Evershed flow corresponds to more negative velocity values). A few cases of large amplitude enhancements of the Evershed signal are associated with temporal enhancements of the intensity: for filament $A$ at $t \approx 24 \mathrm{~min}$ and $t \approx 34 \mathrm{~min}$, and for filament $C$ at $t \approx 56 \mathrm{~min}$. Any further examples of a positive correlation are not obvious from the data. 

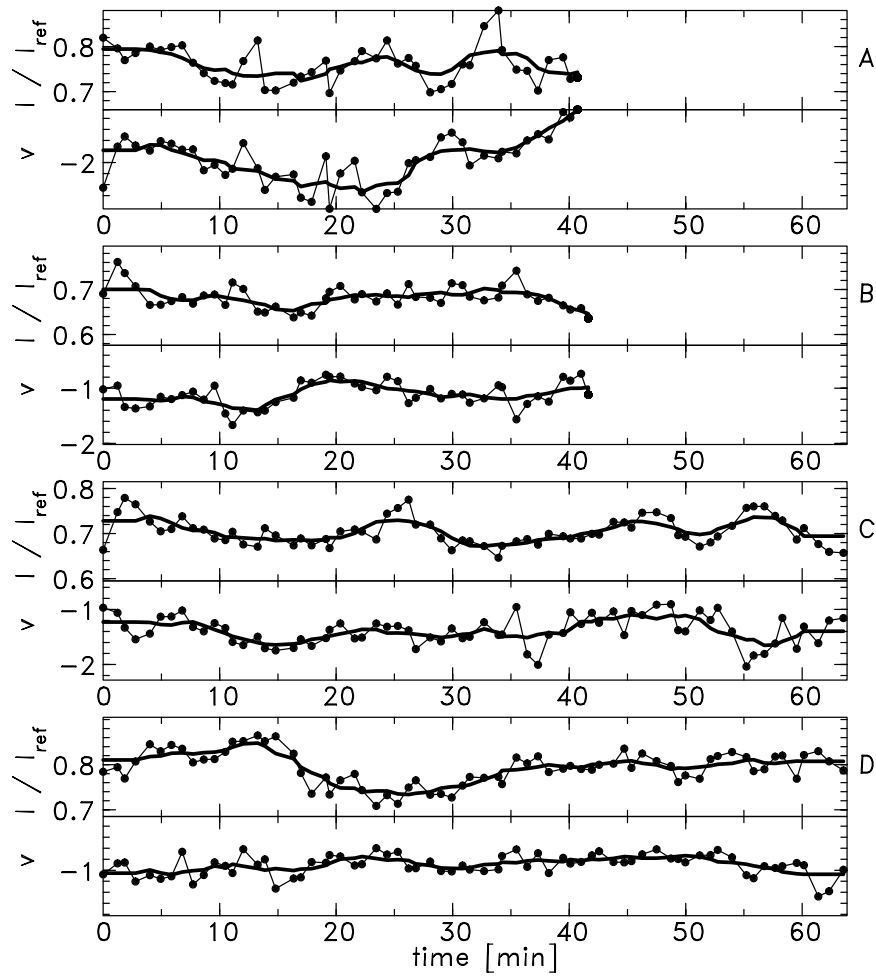

Fig. 6. Comparison of intensity in the far Ca II K wing $(\lambda=3924 \AA)$ and velocity for Mn 3926 for four filaments. Intensity is relative to the mean intensity outside the penumbra.

Given their large amplitude and duration, these few cases of enhanced Evershed signal would likely have been observed as Evershed clouds if a larger surface area had been covered. Though limited in number, these cases support the positive correlation between enhancements of Evershed signal and continuum intensity found by Shine et al. (1994).

\subsection{Velocity signal in bright filaments}

In Fig. 7, the velocity signals for Mn I 3926 in three bright filaments are shown. Like in the dark filaments, the short-time variability is dominated by $p$-mode oscillations. The 5.5-min smoothed data do not suggest any significant variability on longer time scales.

Note the large mean velocity offset of filament $K$ as compared to $M$ and $L$. The orientation of filament $K$ is at low inclination angle with the observation angle so the velocity offset can be attributed to the Evershed effect. This illustrates that the brightness/velocity relationship in the penumbra, the Evershed flow being found predominantly in dark filaments, is not perfect (see also Sects. 3.3 and 4.1).

Penumbral grains are known to migrate through the penumbra, the bulk moving towards the umbra (Muller 1973; Sobotka et al. 1999; Sobotka \& Sütterlin 2001). From the slit-jaw and G-band movies, one clear example of a penumbral grain crossing the slit starting was found. This event can be identified in the intensity profile for filament $L$ in Fig. 7 as an intensity increase starting at $t \approx 30 \mathrm{~min}$. No particular change in the velocity profile is associated with this event, which is rather
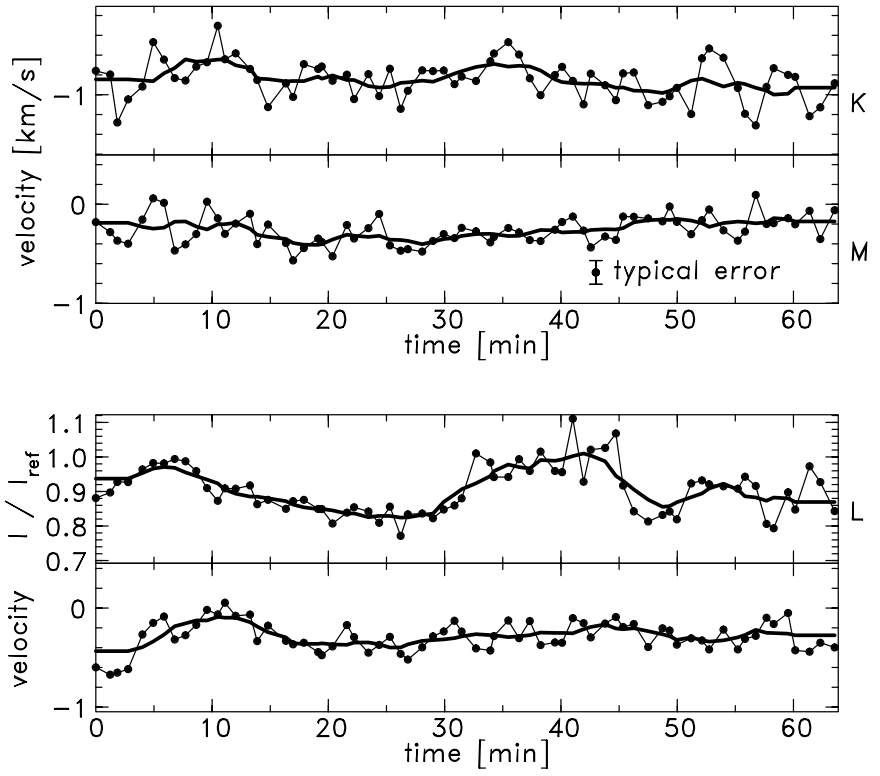

Fig. 7. Velocity signal for Mn 3926 in bright filaments $K, L$ and $M$, plus intensity signal in filament $L$.

unexpected considering reports of systematic upflows in penumbral grains of order 100-200 $\mathrm{m} \mathrm{s}^{-1}$ (Rimmele 1995) and $600 \mathrm{~m} \mathrm{~s}^{-1}$ (Schmidt \& Schlichenmaier 2000).

\section{Summary and discussion}

The time variability of the Evershed effect was studied from high-spatial resolution spectrograms in the wings of the $\mathrm{Ca}$ II $\mathrm{K}$ line combined with Ca II K slit-jaw images and G-band filtergrams. Compared to observations with tunable filter instruments, a clear disadvantage of spectrograph observations is the limited total area that is covered by the spectrograph slit. In this study, this resulted in a statistically rather limited number of filaments being covered and analysed. However, notable advantages of a spectrograph are the seeing-independent and accurate line profiles that can be obtained and the direct access to a series of diagnostic spectral lines.

The results of this study can be summarized as follows:

1. The Evershed flow is found to show irregular variations on a time scale of 8-14 min. This confirms the observation reported by Shine et al. (1990), Shine et al. (1994) and Rimmele (1994).

2. The largest amplitudes in the variation seems to be found where the Evershed flow is strongest: in the deeper parts of the atmosphere and in the strongest channels.

3. There does not seem to be a systematic height dependence for the propagation of the velocity variations.

4. Several examples are found of an Evershed signal enhancement associated with an enhancement in the intensity. This supports the positive correlation between enhancements of Evershed signal and continuum intensity reported by Shine et al. (1994).

5. No significant variability is found in the (weak) Evershed signal detected in bright filaments. 
Currently, no theoretical model incorporates the observed time variability of the Evershed effect in a satisfactory way. Montesinos \& Thomas (1997) remark that siphon flows on the Sun are transient in nature because of the changing conditions at the footpoints. For magnetic loops carrying siphon flows that have one footpoint located outside the penumbra, the conditions on the downstream end of the loop are likely to be dominated by the granulation. In this context, the observed time scale of variability of the Evershed effect is in agreement with the life time of the granules. If the variation in the Evershed effect is driven from the footpoints, the radially outward moving Evershed clouds, as observed by Shine et al. (1994) and Rimmele (1994), indicate that the upstream footpoint is dominant in this process. A disturbance generated at the downstream footpoint would be observed to travel in the upstream direction - contrary to what is observed.

The time scales of the flow tubes in the moving-tube model of Schlichenmaier et al. (1998b) are set by the time scale of interchange convection in their simulations, which is on the order of an hour. Interchange convection of penumbral filaments is unlikely to drive the observed variations; the observed life time of filaments is much longer than the variation time scale (see also Rimmele 1994).

A number of observations have indicated that the bulk of the Evershed flow is concentrated in the lower parts of the atmosphere, at heights below $200 \mathrm{~km}$ (see e.g. Maltby 1964; Schlichenmaier \& Schmidt 2000, Paper I). In Paper I, the line-core Dopplershifts for a number of spectral lines, including the four lines used in this study, were computed from a penumbral atmosphere with a typical Evershed flow channel located at varying heights. This channel was found to account for a $1 \mathrm{~km} \mathrm{~s}^{-1}$ Dopplershift increase for Mn I 3926 when being raised from 40 to $110 \mathrm{~km}$ height (see Fig. 18 of Paper I). The Dopplershift of the other three spectral lines increase with a lesser amount during such a rise since their contribution functions to the line-core intensity peak at greater heights. This illustrates that a slowly rising and sinking Evershed channel could be an alternative explanation of the observed variability in the Evershed effect. As long as the channel stays at heights below the peak of the Mn 3926 contribution function, a rising channel would give a coherent increasing Dopplershift to all four lines. The flow speed does not necessarily have to increase to give rise to larger line-core Dopplershifts. Moving kinks in the Evershed channel, localized elevations in the flow channel, could explain the moving Evershed clouds. In this context it is interesting to note that in the latest simulations of the moving tube model (Schlichenmaier 2002), the flow channel is no longer horizontal (as in the earlier simulations) but develops a wave-like form with moving crests. For this scenario to work, the optical thickness of such channel has to be limited, since an optically thick rising channel would just introduce a geometrical shift of the whole line-forming part of the atmosphere. The continuum level and contribution functions are coherently shifted and no significant change in the line-core Dopplershift could be detected. Obviously, detailed hydrodynamical simulations with a realistic treatment of the radiative transfer are needed to test if such scenario is compatible with the observations. More generally, such detailed modelling is required to get a complete understanding of the Evershed flow embedded in the complex geometry of the sunspot penumbra.

Acknowledgements. The author wishes to thank D. Kiselman for comments on the manuscript. P. Sütterlin is thanked for providing the reduced G-band observations from the Dutch Open Telescope (DOT). The DOT is funded by Utrecht University, the Netherlands Graduate School for Astronomy (NOVA) and the Netherlands Organization for Scientific Research (NWO). The DOT is operated from the Swedish solar telescope building on La Palma. The Swedish Vacuum Solar Telescope (SVST) was operated on the island of La Palma by the Royal Swedish Academy of Sciences in the Spanish Observatorio del Roque de los Muchachos of the Instituto de Astrofísica de Canarias.

\section{References}

Balthasar, H., Sütterlin, P., \& Collados, M. 2001, Astron. Nachr., 322, 367

Beckers, J. M. 1968, Sol. Phys., 3, 258

Evershed, J. 1909, MNRAS, 69, 454

Hammerschlag, R. H., \& Bettonvil, F. C. M. 1998, New Astron. Rev., 42,485

Hirzberger, J., \& Kneer, F. 2001, A\&A, 378, 1078

Johannesson, A. 1993, A\&A, 273, 633

Lites, B., Scharmer, G., \& Skumanich, A. 1990, ApJ, 355, 329

Maltby, P. 1964, Astrophysica Norvegica, 8, 205

Maltby, P. 1997, in Advances in Physics of Sunspots, ASP Conf. Ser., 118, 91

Montesinos, B., \& Thomas, J. H. 1997, Nature, 390, 485

Muller, R. 1973, Sol. Phys., 29, 55

Muller, R. 1992, in Sunspots, Theory and Observations, NATO ASIC Proc., 375, 175

Rimmele, T. 1994, A\&A, 290, 972

Rimmele, T. 1995, A\&A, 298, 260

Rouppe van der Voort, L. H. M. 2002, A\&A, 389, 1020

Scharmer, G., \& Löfdahl, M. 1991, Adv. Space Res., 11, 129

Scharmer, G. B., Pettersson, L., Brown, D. S., \& Rehn, J. 1985, Appl. Opt., 24, 2558

Schlichenmaier, R. 2002, Astron. Nachr., 323, 303

Schlichenmaier, R., Jahn, K., \& Schmidt, H. U. 1998a, ApJ, 493, L121

Schlichenmaier, R., Jahn, K., \& Schmidt, H. U. 1998b, A\&A, 337, 897

Schlichenmaier, R., \& Schmidt, W. 2000, A\&A, 358, 1122

Schmidt, W., \& Schlichenmaier, R. 2000, A\&A, 364, 829

Shand, M., Wei, W., \& Scharmer, G. 1995, in Proc. SPIE 2607, ed. J. Schewel, 145

Shine, R., Smith, K., Tarbell, T., Title, A., \& Scharmer, G. 1990, BAAS, 22, 878

Shine, R., Title, A., Tarbell, T., et al. 1994, ApJ, 430, 413

Shine, R. A. 1990, ANA: http://ana.lmsal.com/

Sobotka, M., Brandt, P., \& Simon, G. 1999, A\&A, 348, 621

Sobotka, M., \& Sütterlin, P. 2001, A\&A, 380, 714

St. John, C. E. 1913a, ApJ, 37, 322

St. John, C. E. 1913b, ApJ, 38, 341

Thomas, J. H. 1994, in NATO ASIC Proc. 433, Solar Surface Magnetism, 219

Title, A., Frank, Z., Shine, R., et al. 1993, ApJ, 403, 780

Wiehr, E. 1999, in Magnetic Fields and Oscillations, ASP Conf. Ser., 184, 86

Wiehr, E., \& Stellmacher, G. 1989, A\&A, 225, 528 